\title{
Utilización de la teoría de Kubelka-Munk para optimizar el reciclado de residuos crudos de gres porcelánico
}

\author{
S. MESTRE", E. SANCHEZ , J. GARCIA ${ }^{*}$, J. SANCHEZ ${ }^{* *}$, C. SOLER ${ }^{* *}$, J. PORTOLES ${ }^{* *}$, J. SALES ${ }^{* *}$ \\ *Instituto de Tecnología Cerámica-AICE. Castellón. \\ **Taulell, S.A.
}

\begin{abstract}
El proceso de fabricación del gres porcelánico genera unos fangos residuales, cuyo vertido presenta problemas de caracter medioambiental. Una posible solución es reintroducir dichos fangos en el proceso productivo, aunque ello conlleva la modificación del color en cocido del producto al que se incorpora. En este trabajo se describe la utilización de la teoría de KubelkaMunk para predecir el color en cocido de la mezcla final, procedimiento que puede optimizar en gran medida el reciclado, ya que permite obtener un material con unas características cromáticas prácticamente idénticas a las de un modelo de baldosa de referencia, de modo que el reciclado de los fangos se pueda llevar a cabo sin que implique alteraciones en el programa de producción.
\end{abstract}

Palabras clave: Gres porcelánico, reciclado, predicción de color, teoría de Kubelka-Munk.

Use of the Kubelka-Munk theory for optimising green porcelain tile waste recycling.

The porcelain tile manufacturing process gives rise to waste sludges, whose disposal entails environmental problems. A possible solution is reuse in the manufacturing process, though this leads to a change of colour in the resulting fired product. The present study describes the use of the Kubelka-Munk theory for predicting the fired colour of the final mixture. This procedure could greatly optimise recycling, since it enables producing a material with chromatic characteristics practically identical to those of the reference tile, so that the sludge can be recycled without altering the production programme

Key words: Porcelain tile, recycling, colour prediction, Kubelka-Munk theory.

\section{INTRODUCCIÓN}

\subsection{Residuos de la fabricación de gres porcelánico}

El gres porcelánico es un pavimento cerámico de muy baja porosidad, obtenido a partir de una mezcla compuesta principalmente de arcillas blancas, cuarzo y feldespatos, molturada vía húmeda, secada por atomización, conformada por prensado y cocida a elevada temperatura $\left(1180-1200^{\circ} \mathrm{C}\right)$. Con fines decorativos generalmente se incluye en la mezcla pigmentos de naturaleza inorgánica (1).

A lo largo de las etapas previas a la cocción se genera un conjunto de residuos formados por las mezclas de materias primas correspondientes a los diferentes modelos o productos cuya elaboración se realiza simultáneamente en la planta (restos de polvo atomizado, baldosas crudas defectuosas o rotas, etc). Debido al gran volumen que pueden alcanzar dichos residuos, alrededor de un $10 \%$ del total de materias primas consumidas (en una planta media significan 10.000 Tm anuales), no es posible realizar un vertido incontrolado de los mismos, y su gestión ha de llevarse a cabo con todas las garantías medioambientales.

Una alternativa al vertido controlado es el reciclado de estos fangos dentro del proceso productivo, ya que su com- posición corresponde a una mezcla de materias primas para obtener gres porcelánico, si bien incorpora los diferentes pigmentos utilizados en la decoración de las baldosas. Por ello, su color en cocido es grisáceo y no coincide exactamente con ningún modelo fabricado, por lo que su reciclado directo no es factible, dado que modificaría las características cromáticas de la baldosa a la que se incorporase.

La solución más realista es utilizar los residuos como un ingrediente de la mezcla de materias primas destinada a la obtención de un modelo de baldosa concreto, que se fabrique en grandes volúmenes para asegurar el reciclado total de los mismos. En este caso el principal problema es determinar la composición de dicha mezcla, para asegurar que el color final en cocido estará dentro de las tolerancias de producción, y sobre todo, obtener un método sistemático para definir dicha composición, sin tener que recurrir a innumerables pruebas, que dependan de la experiencia del operario encargado de la tarea. En este punto es necesario disponer de un modelo teórico que permita predecir el color de las mezclas con buena aproximación, a partir de un número limitado de experimentos. 
En este trabajo se ha analizado la posibilidad de reciclar lotes de residuo de una planta industrial de gres porcelánico, para obtener dos modelos diferentes de baldosas de color gris (referencias G1 y G2). El procedimiento está basado en la teoría de Kubelka-Munk para estimar el color de las mezclas, de modo que se eviten innumerables tanteos. También incluye la utilización de diferentes aditivos para ajustar las coordenadas cromáticas del producto cocido. Los posibles aditivos pueden ser un pigmento negro para oscurecer el color, y circón o una mezcla de materias primas de elevado grado de blancura en cocido, para aclararlo.

\subsection{Predicción del color. Teoría de Kubelka-Munk}

La sensación de color es el resultado de la interpretación que hace el cerebro humano de la radiación visible recogida por los ojos, procedente de los objetos cuando se iluminan con una fuente de luz . La relación entre la energía luminosa incidente y la devuelta por la superfice del cuerpo para cada longitud de onda es la curva de reflectancia, $R=f(\lambda)$, que caracteriza el color del mismo (2).

Se han propuesto diferentes sistemas de coordenadas cromáticas para describir el color de los objetos, de los cuales el que ha alcanzado mayor difusión en el campo de la cerámica es el sistema CIELAB (3). Sin embargo, en dicho sistema no existe una relación directa entre los valores de $L^{*}, a^{*}$ y b* con las proporciones de los diferentes ingredientes que forman parte de un material cerámico (ya sea un vidriado o una composición cerámica), si bien se han propuesto algunas aproximaciones simples para estimar la composición de una mezcla destinada a obtener un color en cocido determinado, a partir de las coordenadas cromáticas de los ingredientes (4).

La predicción del color de una mezcla, cuando diversos pigmentos se adicionan a un material base, se puede realizar a partir de la teoría de Kubelka-Munk (5) . Dicha teoría ha sido utilizada en campos tan diversos como los plásticos (6) y los vidriados cerámicos (7), bajo determinadas condiciones.

La teoría de Kubelka-Munk está basada en el supuesto de que el comportamiento óptico de un material que absorbe y dispersa la radiación visible de una longitud de onda determinada, puede ser descrito por dos constantes, el coeficiente de absorción K, y el coeficiente de dispersión S. En el caso de que el material esté formado por varios componentes, sus coeficientes de absorción y dispersión vendrán dados por los respectivos coeficientes de cada componente, multiplicados por las proporciones de los mismos en la mezcla.

$$
\mathrm{K}_{\mathrm{m}}=\sum_{\mathrm{i}=1}^{\mathrm{n}} \mathrm{w}_{\mathrm{i}} \cdot \mathrm{K}_{\mathrm{i}} \quad \mathrm{S}_{\mathrm{m}}=\sum_{\mathrm{i}=1}^{\mathrm{n}} \mathrm{w}_{\mathrm{i}} \cdot \mathrm{S}_{\mathrm{i}}
$$

Siendo $\mathrm{n}$ el número de componentes en la mezcla y w las fracciones másicas de los mismos.

Para hacer uso de esta teoría es necesario conocer los valores de los coeficientes K y S. La relación entre dichos coeficientes y la reflectancia a cada longitud de onda viene dada por la ecuación [2], que es una modificación de la fórmula original de Kubelka-Munk, la cual incorpora ciertas correcciones no contempladas por los autores en su teoría inicial $(8,9)$.

$$
\frac{\mathrm{K}}{\mathrm{S}}=\frac{0.45(1-\mathrm{R})^{2}}{\mathrm{R}^{2}+1.36 \mathrm{R}-0.056}
$$

Esta expresión permite calcular directamente los valores del cociente entre $\mathrm{K}$ y S para cualquier material en función de los valores de la reflectancia, para cada longitud de onda (la reflectancia estará calculada en tanto por uno). Por tanto, si se conocen los valores de $K=f(\lambda)$ y $S=f(\lambda)$ de todos los componentes de un material, es posible calcular los valores de $\mathrm{K}_{\mathrm{m}} \mathrm{y}$ $\mathrm{S}_{\mathrm{m}}$ para el conjunto a partir de la composición del mismo (ecuación [1]), y con la expresión [2] determinar la reflectancia a cada longitud de onda. A partir de dicha curva de reflectancia se calculan las coordenadas cromáticas del material (10), de modo que esta teoría permite estimar el color de un material, a partir de datos característicos de sus componentes.

La principal dificultad para emplear esta teoría es que a partir de las curvas de reflectancia, que son los datos físicos disponibles en medidas realizadas con espectrofotómetros, no se pueden calcular por separado K y S, ya que sólo se puede obtener el valor de su cociente. Por otra parte, la medida directa de $\mathrm{K}$ y $S$ en base a la teoría de Kubelka-Munk resulta experimentalmente muy compleja ya que en materiales opacos es necesario disponer de láminas de espesor conocido, y suficientemente reducido para que al realizar medidas de reflectancia sobre fondo negro y fondo blanco, se obtengan valores diferentes (11).

Una alternativa para resolver el problema es introducir en la mezcla un ingrediente del cual se conozca al menos uno de sus coeficientes, o bien se le puede asignar un valor arbitrario, pero coherente con su comportamiento. A partir de mezclas de distintas proporciones de dicho material de referencia con el componente básico de la mezcla (el que interviene en mayor proporción), se pueden calcular los valores de $\mathrm{K}$ y S de dicho componente básico, ya que para cada longitud de onda se obtendrá un sistema con tantas ecuaciones del tipo [3], como mezclas se hayan preparado, y cuyas curvas de reflectancia se habrán medido experimentalmente.

$$
\left(\frac{K}{S}\right)_{j}=\frac{K_{R} \cdot w_{R}+K_{B} \cdot w_{B}}{S_{R} \cdot w_{R}+S_{B} \cdot w_{B}}
$$

Donde:

$K_{R}$ y $S_{R}$ son los coeficientes del material de referencia.

$\mathrm{K}_{\mathrm{B}}$ y $_{\mathrm{B}}$ son los coeficientes del material base de la composición.

$\mathrm{W}_{\mathrm{R}} \mathrm{y}_{\mathrm{B}}$ son las fracciones másicas de los materiales de referencia y base de la composición.

$(\mathrm{K} / \mathrm{S})_{\mathrm{j}}$ es el cociente de los coeficientes $\mathrm{K}$ y $\mathrm{S}$ para la mezcla $\mathbf{j}$, de material base con el de referencia en proporciones $\mathrm{W}_{\mathrm{B}}$ $\mathrm{y} \mathrm{w}_{\mathrm{R}^{\prime}}$ calculado a partir de la curva de reflectancia.

Supuesto que se conoce o se asigna el valor de uno de los coeficientes del material de referencia, por ejemplo $S_{R}=f(\lambda)$, son necesarios al menos tres datos de $K / S$ experimentales (correspondientes al material base puro y dos mezclas en proporciones diferentes con el material de referencia), para calcular los otros tres coeficientes que aparecen en la ecuación. Si se dispone de un número de mezclas superior a dos $(n>2)$, se puede realizar una optimización sobre los valores de $\mathrm{K}$ y S, para minimizar las diferencias entre los valores experimentales de la reflectancia para cada longitud de onda y cada mezcla, y los calculados con las ecuaciones [3] y [2] a partir de los valores individuales de K y S, según la expresión:

$$
\sum_{j=1}^{n}\left[R\left(w_{B}^{j}\right)_{\exp }-R\left(K_{R}, S_{B}, K_{B}, w_{B}^{j}\right)\right]^{2}=\text { mínimo }
$$

Una elección aceptable como material de referencia en el caso del gres porcelánico, es un opacificante que presente una reactividad muy reducida con la fase vítrea generada durante 
la cocción, a cuyo coeficiente de dispersión se le puede asignar un valor unidad para todas las longitudes de onda del espectro visible $\left(S_{R}=1 \neq f(\lambda)\right)$. Por ello en este trabajo se escogió el circón como material de referencia, ya que es un opacificamente ampliamente utilizado en la industria cerámica en general, y en la fabricación de gres porcelánico en particular. Además es uno de los ingredientes escogidos para blanquear el color en cocido de las mezclas si fuera necesario.

Una vez conocidos los valores de los coeficientes K y S para el material base, se pueden determinar los coeficientes $\mathrm{K}$ y S para el resto de ingredientes de la mezcla, preparando mezclas binarias del material base con cada uno de los ingredientes y aplicando el mismo procedimiento de cálculo utilizado con el material de referencia. Es decir, se dispondrá de un sistema de tantas ecuaciones del tipo [3], como mezclas del material base con cada componente concreto $A_{i}$ que se hayan realizado.

Una vez se dispone de los datos de $K_{i}=f(\lambda)$ y $S_{i}=f(\lambda)$ de todos los posibles ingredientes, ya es factible estimar las curvas de reflectancia en cocido de las mezclas que se puedan plantear en base a las ecuaciones desarrolladas, que se pueden resumir como:

$$
\frac{\sum_{\mathrm{i}=1}^{\mathrm{n}} \mathrm{K}_{\mathrm{i}} \cdot \mathrm{w}_{\mathrm{i}}}{\sum_{\mathrm{i}=1}^{\mathrm{n}} \mathrm{S}_{\mathrm{i}} \cdot \mathrm{w}_{\mathrm{i}}}=\left(\frac{\mathrm{K}}{\mathrm{S}}\right)_{\mathrm{m}}=\frac{0.45\left(1-\mathrm{R}_{\mathrm{m}}\right)^{2}}{\mathrm{R}_{\mathrm{m}}^{2}+1.36 \mathrm{R}_{\mathrm{m}}-0.056}
$$

A partir de esta expresión, conocido $(\mathrm{K} / \mathrm{S})_{\mathrm{m}}$ se pueden despejar los valores de la reflectancia de la mezcla para cada longitud de onda $\left(R_{m}\right)$, y a partir de dichos datos obtener las coordenadas cromáticas.

El procedimiento de cálculo descrito se utiliza dentro de un ciclo iterativo, donde se calculan las coordenadas cromáticas de diferentes mezclas, con el fin de minimizar la diferencia de color entre el material a reproducir (por ejemplo: el modelo de baldosa G2), y la mezcla de materias primas disponibles (por ejemplo: un residuo y pigmento negro), hasta conseguir que el $\Delta \mathrm{E}^{*}$ entre la composición industrial que se desea reproducir y la calculada a partir de los valores de $K_{i} y$ $S_{\mathrm{i}}$, para los distintos ingredientes, sea mínima. El diagrama de flujo del proceso de cálculo se describe en la figura 1.

En el cálculo de los porcentajes en peso de cada componente en el producto cocido, se ha contemplado la pérdida por calcinación que sufren los residuos durante la cocción, mientras que en el caso del circón y el pigmento negro, se ha considerado que estos materiales no sufren una pérdida de peso apreciable.

\section{EXPERIMENTAL}

\subsection{Materiales}

El estudio se ha realizado con dos muestras de residuo (referencias R1 y R2), obtenidas en planta durante diferentes periodos. Los materiales cuyo color se deseaba reproducir fueron dos modelos de baldosa de color gris de diferente tono (referencias G1 y G2). Como aditivos para modificar el color se utilizaron circón $\left(\mathrm{D}_{50}=1.7 \mu \mathrm{m}\right)$ y un pigmento negro de cobalto industrial, siendo ambos materiales habitualmente utilizados en la producción de baldosas de gres porcelánico. También se realizaron ensayos con una composición de gres porcelánico de elevada blancura, pero los cálculos demostraron que no presentaba ninguna ventaja con respecto al uso del circón.

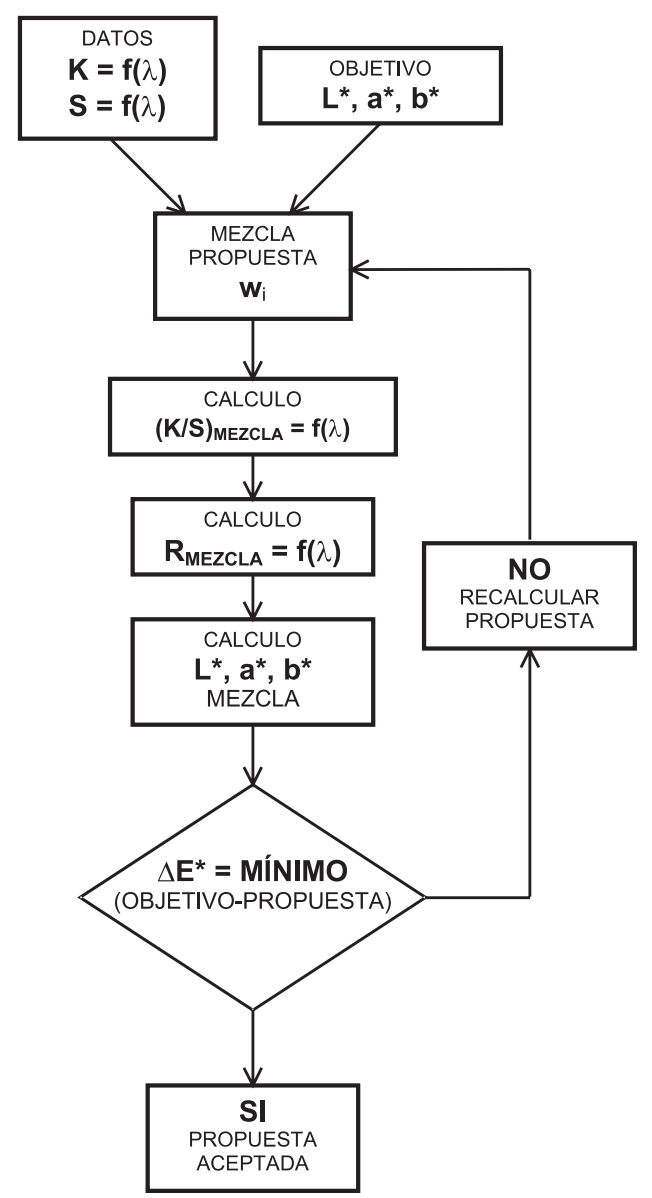

Figura 1. Diagrama de flujo del proceso de cálculo de la mezcla de materias primas cuyo color en cocido es lo más próximo posible al del producto industrial que se desea reproducir.

\subsection{Procedimiento}

Las mezclas de materias primas correspondientes a las baldosas industriales (G1 y G2), se suministraron en forma de polvo atomizado, y su caracterización se realizó sin someterlas a ningún tratamiento previo. Los residuos se suministraron en forma de terrones de material seco y su caracterización se realizó tras desleirlos previamente en un dispersor de alta intensidad durante 5 minutos utilizando una mezcla acetonaagua como medio de dispersión (100 g de sólido, $190 \mathrm{ml}$ de acetona y $10 \mathrm{ml}$ de agua). La suspensión obtenida se secó bajo lámparas de infrarrojos. Las mezclas residuo+aditivos se prepararon desleyendo los componentes en el dispersor de alta intensidad, en las mismas condiciones que las utilizadas para los residuos. Las suspensiones obtenidas se secaron bajo lámparas de infrarrojos.

Tras el secado, el polvo seco se tamizó en una malla de 500 $\mu \mathrm{m}$ para evitar la presencia de aglomerados de gran tamaño. Los polvos de prensa se humectaron hasta un contenido en agua del 5.5\% (base seca), y posteriormente se prensaron en una prensa automática de laboratorio a la presión adecuada en cada caso para conseguir una densidad aparente en seco de $1.91 \mathrm{~g} / \mathrm{cm}^{3}$. Las probetas se secaron en una estufa de laboratorio a $110^{\circ} \mathrm{C}$ un mínimo de tres horas.

Las probetas secas se cocieron en un horno eléctrico de laboratorio con un ciclo estándar de 50 minutos y un tiempo de permanencia a temperatura máxima de 6 minutos. 
En el caso de las probetas destinadas al estudio colorimétrico (polvo atomizado industrial, residuos y mezclas residuo+aditivos), se sintetizaron doce probetas a la temperatura de máxima densificación, de las que se determinó la curva de reflectancia y las coordenadas cromáticas.

La medida de la curva de reflectancia y las coordenadas cromáticas de las probetas se realizó con un espectrofotómetro de reflectancia difusa Macbeth Color-Eye 7000. Las condiciones de medida del equipo fueron: Iluminante Estándar CIE C, Observador Estándar CIE $2^{\circ}$, componentes ultravioleta y especular incluidas. A partir de las medidas del conjunto de probetas, se determinó la curva media de reflectancia, promediando los valores de dicha variable a cada longitud de onda. Asimismo, se calculó el valor medio de cada coordenada cromática.

El cálculo de las coordenadas cromáticas de las mezclas a partir de los valores de reflectancia calculados con el modelo descrito en la introducción, se realizó siguiendo el método especificado en la norma ASTM E-308, haciendo uso de las tablas correspondientes al Iluminante Estándar CIE C y el Observador Estándar CIE $2^{\circ}$.

\section{RESULTADOS Y DISCUSIÓN}

\subsection{Caracterización de los materiales de partida}

Los resultados de la caracterización de las mezclas industriales y de los residuos, cocidos a la temperatura de máxima densificación de los productos industriales $\left(1200^{\circ} \mathrm{C}\right)$, se detallan en la tabla I y Figura2.

Comparando con los colores de las baldosas industriales a reproducir, los residuos son más oscuros, algo más rojizos y menos amarillentos que la G1. En cuanto a la baldosa G2, resulta mucho más oscura y azulada que los residuos.

Aunque los residuos son relativamente similares desde el punto de vista colorimétrico, en comparación con las baldosas industriales, se aprecian diferencias en la forma de sus curvas de reflectancia (figura 2), lo cual puede ejercer una influencia notable sobre el color en cocido de las mezclas que se puedan sintetizar a partir de los mismos.

\subsection{Determinación de los valores de $\mathrm{K}_{\mathrm{i}}$ y $\mathrm{S}_{\mathrm{i}}$ de los diferentes ingredientes}

La obtención de los valores de $K=f(\lambda)$ y $S=f(\lambda)$ para los dos residuos, así como $\mathrm{K}=\mathrm{f}(\lambda)$ para el circón incorporado a los mismos, se realizó siguiendo el procedimiento descrito previamente, y elaborando tres mezclas diferentes (proporciones en peso circón:residuo 1:99, 3:97 y 5:95), además del residuo sin adición, para realizar la optimización numérica (ecuación [4]). Una vez obtenidos los datos, se procedió a calcular los valores de $\mathrm{K}=\mathrm{f}(\lambda)$ y $\mathrm{S}=\mathrm{f}(\lambda)$, correspondientes al pigmento negro incorporado en ambos residuos, a partir de tres mezclas de residuo y pigmento (proporciones en peso pigmento:residuo 1:99, 2:98 y 3:97), además del residuo sin adición.

Los valores de K y S calculados para los dos residuos, así como para el circón introducido en los mismos se detallan en la figura 3, donde se aprecia que las curvas correspondientes a cada residuo son diferentes entre sí, aunque bastante próximas, lo cual es coherente con las diferencias halladas en las curvas de reflectancia.

En el caso del circón, su coeficiente de absorción es muy reducido y regular en los dos residuos, lo cual confirma su
TABla I. Resultados DE LA CARACTERIZACIÓN DE LAS MEZClAS INDUSTRIALES (G1 y G2) Y LOS RESIDUOS (R1 y R2), COCIDOS A $1200^{\circ} \mathrm{C}$.

\begin{tabular}{|l|l|l|l|l|}
\hline Propiedades & G1 & G2 & R1 & R2 \\
\hline Presión de prensado $\left(\mathrm{kg}^{\prime} \mathrm{cm}^{2}\right)$ & 360 & 345 & 395 & 395 \\
Densidad aparente en cocido $\left(\mathrm{g} / \mathrm{cm}^{3}\right)$ & 2.425 & 2.407 & 2.428 & 2.427 \\
Contracción lineal en cocción $(\%)$ & 8.6 & 8.4 & 8.3 & 8.3 \\
Pérdida por calcinación $(\%)$ & 3.27 & 3.33 & 3.63 & 3.56 \\
\hline Coordenada L* & 65.08 & 33.55 & 59.17 & 61.28 \\
Coordenada a* & -0.35 & 0.77 & 0.04 & 0.13 \\
Coordenada b* & 5.21 & -0.55 & 4.04 & 4.95 \\
\hline
\end{tabular}

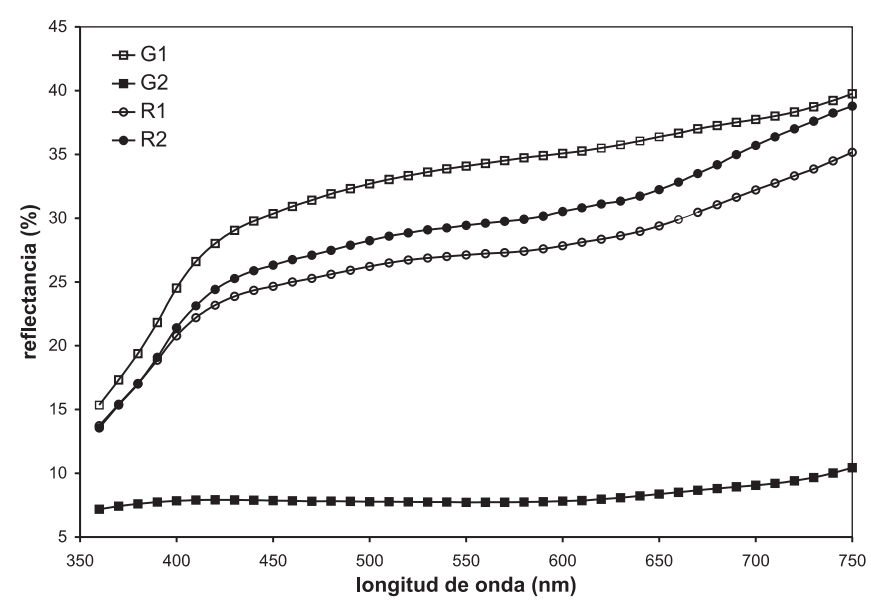

Figura 2. Curvas de reflectancia correspondientes a los productos industriales (G1 y G2), y los residuos (R1 y R2).

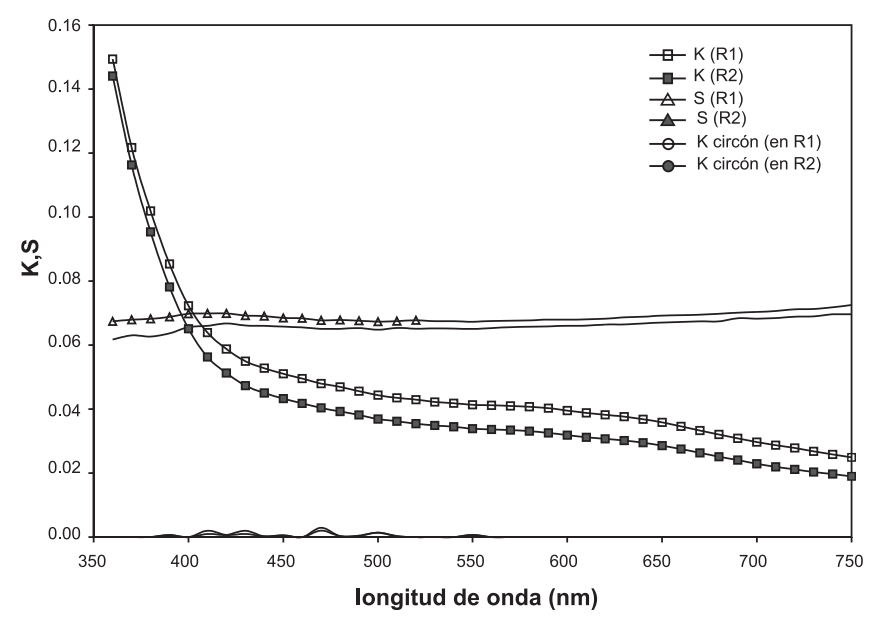

Figura 3. Valores de K y S correspondientes a los dos residuos (R1 y $\mathrm{R} 2)$, así como valores de $\mathrm{K}$ correspondientes al circón, calculados en los dos residuos utilizados.

función de opacificante, sin que modifique la cromaticidad de la mezcla a la que se adiciona. Debido al reducido valor, no se detectan diferencias apreciables entre los valores calculados para cada uno de los residuos.

Los valores de $\mathrm{K}$ y S obtenidos para el pigmento en los dos residuos se detallan en la figura 4 , donde se aprecia que el coeficiente de absorción es muy elevado para todas las longi- 


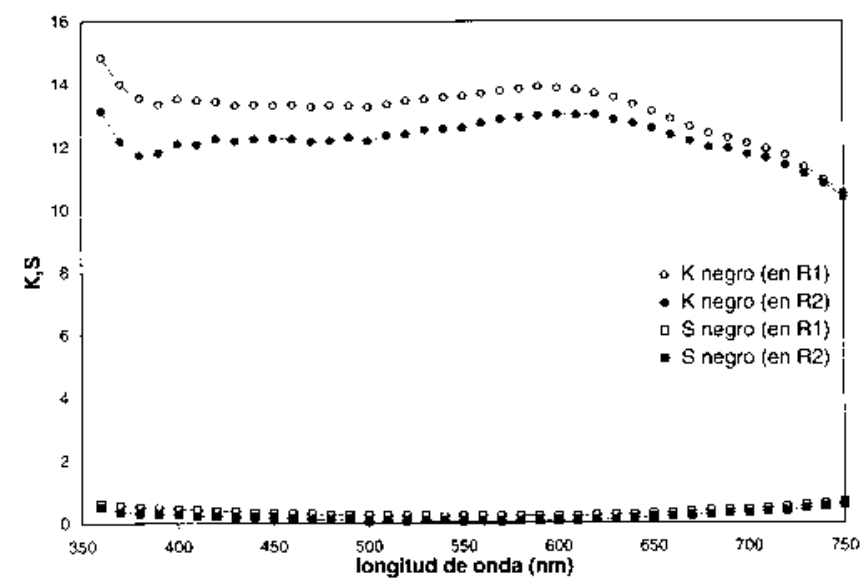

Figura 4. Valores de $\mathrm{K}$ y S correspondientes al pigmento negro, en los dos residuos utilizados.

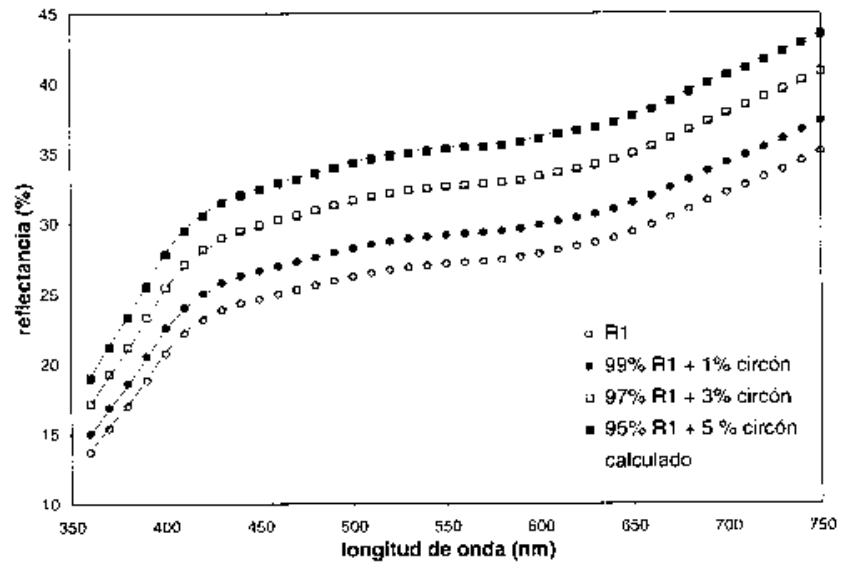

Figura 5. Curvas de reflectancia de las mezclas de residuo R1 y circón, tanto experimentales como obtenidas a partir de los valores de K y S calculados.

tudes de onda del espectro, y el de dispersión bastante más bajo, como corresponde a un pigmento negro. A pesar de utilizarse el mismo pigmento, los valores de $\mathrm{K}$ y S no coinciden, lo cual implica que cada uno de los residuos interacciona de forma diferente con el pigmento. Este mismo hecho impide que se puedan considerar los valores de $\mathrm{K}$ y $\mathrm{S}$ para el pigmento independientes del residuo al que se incorporan, y por lo tanto tendrán que obtenerse experimentalmente para cada lote de residuo que se quiera reciclar.

A partir de los valores de $\mathrm{K}$ y S calculados se obtiene una reconstrucción muy buena de las curvas de reflectancia experimentales (figura 5, figura 6, Figura 7 y Figura 8), lo cual se refleja en las escasas diferencias halladas en los valores de las coordenadas cromáticas (Tabla II). En todos los casos los valores de $\Delta \mathrm{E}^{*}$ entre las coordenadas experimentales y las calculadas son inferiores a 0.35 , que es inferior al umbral de distinción del ojo humano. En consecuencia, los valores de K y S obtenidos se pueden considerar adecuados para el conjunto de ingredientes seleccionados para reproducir el color en cocido de las composiciones industriales de gres porcelánico, G1 y G2.

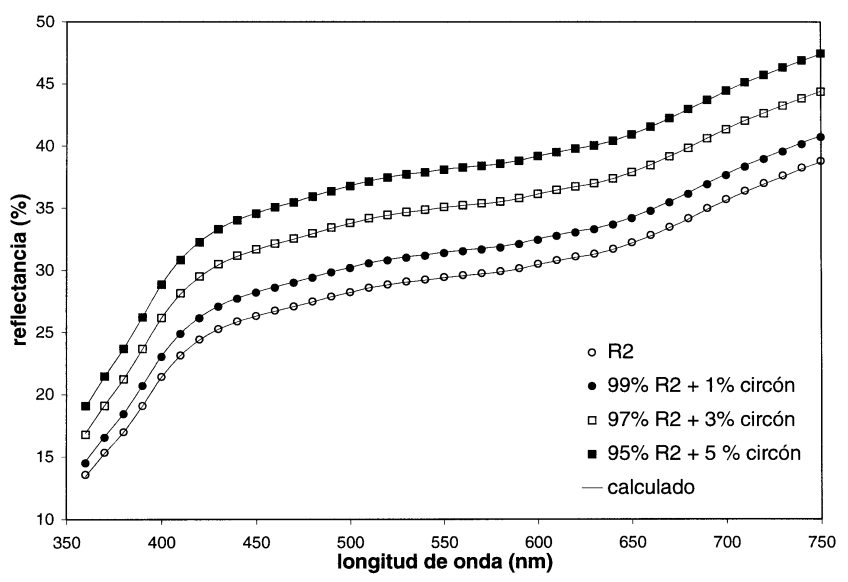

Figura 6. Curvas de reflectancia de las mezclas de residuo R2 y circón, tanto experimentales como obtenidas a partir de los valores de $\mathrm{K}$ y $\mathrm{S}$ calculados.

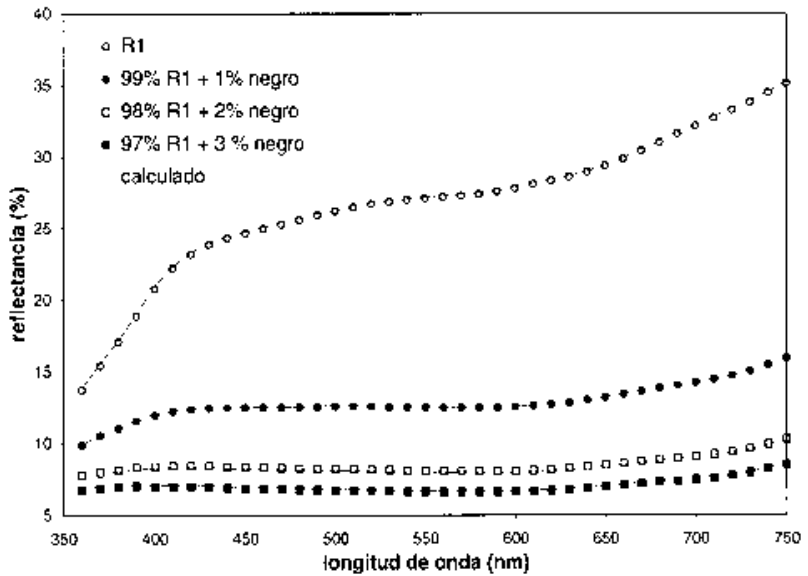

Figura 7. Curvas de reflectancia de las mezclas de residuo R1 y pigmento negro, tanto experimentales como obtenidas a partir de los valores de $\mathrm{K}$ y S calculados.

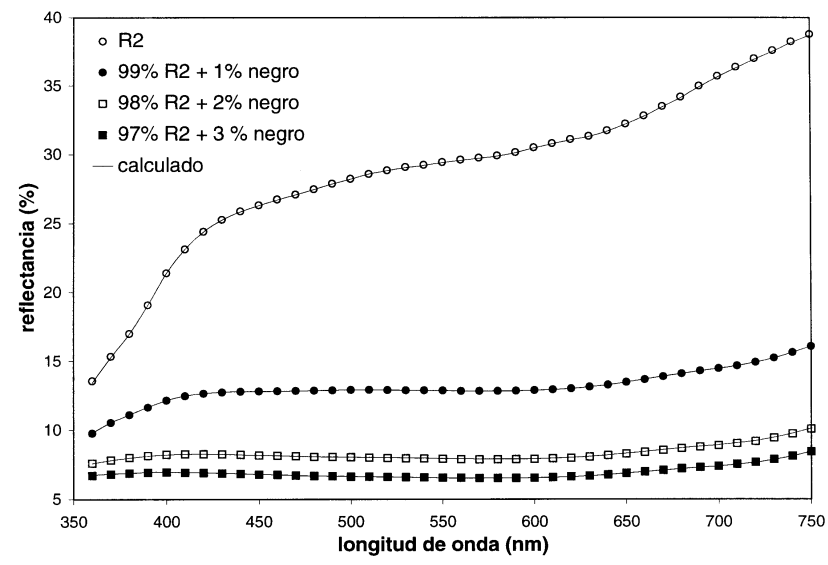

Figura 8. Curvas de reflectancia de las mezclas de residuo R2 y pigmento negro, tanto experimentales como obtenidas a partir de los valores de $\mathrm{K}$ y $\mathrm{S}$ calculados. 
TABla II. COORDENADAS CROMÁTICAS EXPERIMENTALES Y CALCULADAS A PARTIR DE LOS VALORES DE K Y S, PARA LAS DIFERENTES MEZCLAS ELABORADAS.

\begin{tabular}{|c|c|c|c|c|c|c|c||}
\hline \multirow{2}{*}{ Muestras } & \multicolumn{3}{|c|}{ datos experimentales } & \multicolumn{2}{c|}{ datos calculados } & \multirow{2}{*}{$\Delta \mathrm{E}^{*}$} \\
\cline { 2 - 7 } & $\mathrm{L}^{*}$ & $\mathrm{a}^{*}$ & $\mathrm{~b}$ & $\mathrm{~L}^{*}$ & $\mathrm{a}^{*}$ & $\mathrm{~b}^{*}$ & \\
\hline R1 & 59.17 & 0.04 & 4.04 & 59.24 & 0.05 & 4.00 & 0.09 \\
$99 \% \mathrm{R} 1+1 \%$ circón & 61.01 & 0.01 & 3.98 & 60.96 & -0.01 & 4.00 & 0.05 \\
$97 \% \mathrm{R} 1+3 \%$ circón & 63.93 & -0.08 & 3.96 & 63.82 & -0.08 & 3.96 & 0.11 \\
$95 \% \mathrm{R} 1+5 \%$ circón & 66.06 & -0.13 & 3.92 & 66.14 & -0.13 & 3.90 & 0.09 \\
$99 \% \mathrm{R} 1+1 \%$ negro & 42.09 & 0.34 & 0.22 & 42.14 & 0.33 & 0.19 & 0.06 \\
$98 \% \mathrm{R} 1+2 \%$ negro & 34.29 & 0.46 & -0.67 & 34.07 & 0.49 & -0.60 & 0.23 \\
$97 \% \mathrm{R} 1+3 \%$ negro & 31.03 & 0.58 & -0.72 & 31.20 & 0.55 & -0.78 & 0.19 \\
\hline R2 & 61.28 & 0.13 & 4.95 & 61.25 & 0.12 & 4.91 & 0.05 \\
$99 \% \mathrm{R} 2+1 \%$ circón & 62.93 & 0.06 & 4.85 & 62.99 & 0.06 & 4.86 & 0.06 \\
$97 \% \mathrm{R} 2+3 \%$ circón & 65.90 & -0.03 & 4.73 & 65.86 & -0.02 & 4.74 & 0.03 \\
$95 \% \mathrm{R} 2+5 \%$ circón & 68.16 & -0.06 & 4.64 & 68.17 & -0.07 & 4.61 & 0.04 \\
$99 \% \mathrm{R} 2+1 \%$ negro & 42.60 & 0.21 & 0.25 & 42.59 & 0.22 & 0.22 & 0.03 \\
$98 \% \mathrm{R} 2+2 \%$ negro & 33.89 & 0.50 & -0.75 & 33.93 & 0.46 & -0.66 & 0.10 \\
$97 \% \mathrm{R} 2+3 \%$ negro & 30.86 & 0.54 & -0.78 & 30.82 & 0.57 & -0.85 & 0.09 \\
\hline \hline
\end{tabular}

TABLA III. COMPOSICIONES CALCULADAS PARA IGUALAR EL COLOR DE LOS PRODUCTOS INDUSTRIALES A PARTIR DE LOS RESIDUOS, Y COORDENADAS CROMÁTICAS CALCULADAS A PARTIR DE LOS VALORES DE K Y S DE LOS DISTINTOS INGREDIENTES.

\begin{tabular}{|l|c|c|c|c|c|c|c|c|}
\hline \multirow{3}{*}{ producto } & \multicolumn{4}{|c|}{ Composición (\% en peso) } & \multicolumn{4}{c|}{ Datos colorimétricos calculados } \\
\cline { 2 - 9 } & $\mathrm{R} 1$ & $\mathrm{R} 2$ & circón & negro & $\mathrm{L}^{*}$ & $\mathrm{a}^{*}$ & $\mathrm{~b}^{*}$ & $\Delta \mathrm{E}^{*}$ \\
\hline \multirow{3}{*}{ G1 } & 96.00 & - & 4.00 & - & 65.05 & -0.11 & 3.93 & 1.30 \\
\cline { 2 - 9 } & - & 97.60 & 2.40 & - & 65.07 & 0.00 & 4.78 & 0.56 \\
\hline \multirow{3}{*}{ G2 } & 96.75 & - & - & 3.25 & 33.57 & 0.50 & -0.64 & 0.49 \\
\cline { 2 - 9 } & - & 96.84 & - & 3.16 & 33.57 & 0.47 & 0.69 & 0.55 \\
\hline
\end{tabular}

TABLA IV. DATOS COLORIMÉTRICOS DE LAS COMPOSICIONES PREPARADAS PARA REPRODUCIR LOS PRODUCTOS INDUSTRIALES

\begin{tabular}{|l|l|l|l|l|l|l||}
\hline producto & Residuo & $\mathrm{L}^{*}$ & $\mathrm{a}^{*}$ & $\mathrm{~b}^{*}$ & $\Delta \mathrm{E}^{*}{ }_{\mathrm{I}}$ & $\Delta \mathrm{E}^{*}{ }_{\mathrm{II}}$ \\
\hline \multirow{2}{*}{ G1 } & R1 & 64.91 & -0.15 & 3.95 & 1.29 & 0.15 \\
& R2 & 64.92 & 0.06 & 4.72 & 0.66 & 0.17 \\
\hline \multirow{2}{*}{ G2 } & R1 & 33.64 & 0.48 & -0.70 & 0.56 & 0.10 \\
& R2 & 33.05 & 0.53 & -0.65 & 0.70 & 0.52 \\
\hline
\end{tabular}

\subsection{Reproducción de los productos industriales G1 y G2}

Con los datos de $\mathrm{K}$ y $\mathrm{S}$ se procedió a calcular la composición de las mezclas de materias primas necesarias para igualar el color de los productos G1 y G2 a partir de los dos residuos. Los resultados de los cálculos fueron las proporciones de residuo, circón y pigmento negro necesarias en cada caso, así como las coordenadas cromáticas de la mezcla calculada y el $\Delta \mathrm{E}^{*}$ con respecto a los productos industriales (tabla III).

Los cálculos indican que un gres porcelánico de color muy parecido al G2 se podrá obtener a partir de los dos residuos, pero la coloración de la baldosa G1 sólo se puede reproducir a partir del residuo $\mathrm{R} 2$, ya que si se incorpora también el residuo $\mathrm{R} 1$ se obtendría un material cuyo $\Delta \mathrm{E}^{*}$ con respecto al producto industrial sería demasiado elevado.

Las coordenadas cromáticas de las composiciones cal- culadas para reproducir los productos industriales se detallan en la tabla IV, donde se ha calculado tanto la diferencia de color entre el producto industrial y el obtenido en laboratorio $\left(\Delta \mathrm{E}_{\mathrm{r}}^{*}\right)$, como la diferencia entre este último y el color calculado para esa misma mezcla de materias primas $\left(\Delta \mathrm{E}_{\mathrm{II}}^{*}\right)$. En las figuras 9 y 10 se comparan las curvas de reflectancia de los productos G1 y G2, con las calculadas a partir de los valores de $\mathrm{K}$ y S, y las obtenidas experimentalmente con las mezclas diseñadas para reproducir los productos industriales.

Los resultados indican que la predicción realizada con la teoría de Kubelka-Munk es bastante precisa, ya que en todos los casos se consigue predecir el color de la composición propuesta con un $\Delta \mathrm{E}^{*}$ inferior a la unidad $\left(\Delta \mathrm{E}_{\mathrm{II}}^{*}\right)$, y además las diferencias con respecto a los productos industriales son del 


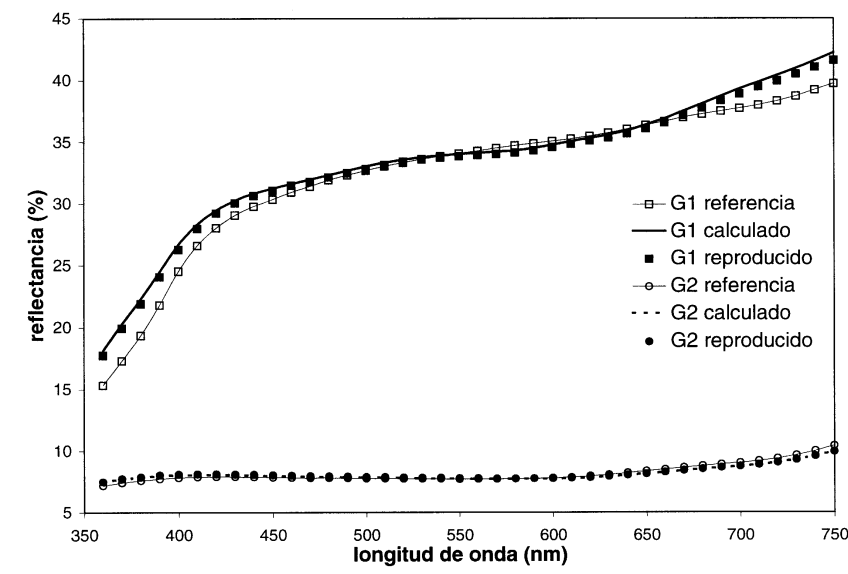

Figura 9. Comparación entre las curvas de reflectancia correspondientes a los productos industriales (G1 y G2 referencia), las calculadas a partir de los valores de $\mathrm{K}$ y $\mathrm{S}$ para las composiciones óptimas estimadas para igualarlos a partir del residuo R1 (G1 y G2 calculado), y las correspondientes a la preparación en laboratorio de las composiciones que reproducen los productos industriales (G1 y G2 reproducido).

mismo orden que las estimadas $\left(\Delta \mathrm{E}_{\mathrm{I}}^{*}\right)$. También, se confirma que no es posible reproducir el producto G1 empleando el residuo R1, ya que la diferencia de color entre el producto industrial y el obtenido con la composición que minimiza $\Delta \mathrm{E}^{*}$, es superior a la unidad.

Los valores obtenidos para $\Delta \mathrm{E}_{\mathrm{I}}^{*}$ están directamente relacionados con las diferencias entre las curvas $R=f(\lambda)$ de los productos que se desea reproducir (G1 y G2), y las curvas correspondientes a los residuos utilizados (R1 y R2). Si dichas curvas poseen una forma muy diferente, resulta imposible conseguir a partir de un residuo una mezcla cuya curva $R=f(\lambda)$ en cocido sea similar a la del producto industrial que se desea reproducir, adicionando un opacificante o un pigmento negro. Este es el caso del producto G1, que es posible reproducirlo con bastante aproximación a partir del residuo $\mathrm{R} 2$, ya que las diferencias en la curva $R=f(\lambda)$ sólo aparecen en la zona del espectro correspondiente a las longitudes de onda más elevadas (figura 10), mientras que resulta imposible reproducirlo a partir del residuo R1 porque en dicho caso las diferencias aparecen tanto en longitudes de onda cortas como largas (figura 9).

Un procedimiento para reducir estas diferencias en las curvas $R=f(\lambda)$ podría ser la utilización de pigmentos que puedan modificar la forma de dicha curva (ej un azul para reducir la reflectancia a longitudes de onda elevadas), a diferencia de opacificantes y pigmentos negros cuyo principal efecto es desplazar la curva de modo relativamente paralelo a sí misma. Sin embargo ello complicaría mucho el problema, debido a que sería imprescindible calcular los valores de $\mathrm{K}$ y S para cada uno de los posibles pigmentos a incorporar. Una alternativa más simple es realizar una gestión adecuada de los diferentes residuos que se producen en la planta, combinando materiales de diferente color de cocción, para conseguir que cada lote de residuo tenga una tonalidad neutra, o a lo sumo ligeramente parecida a la de los productos que se desea reproducir.

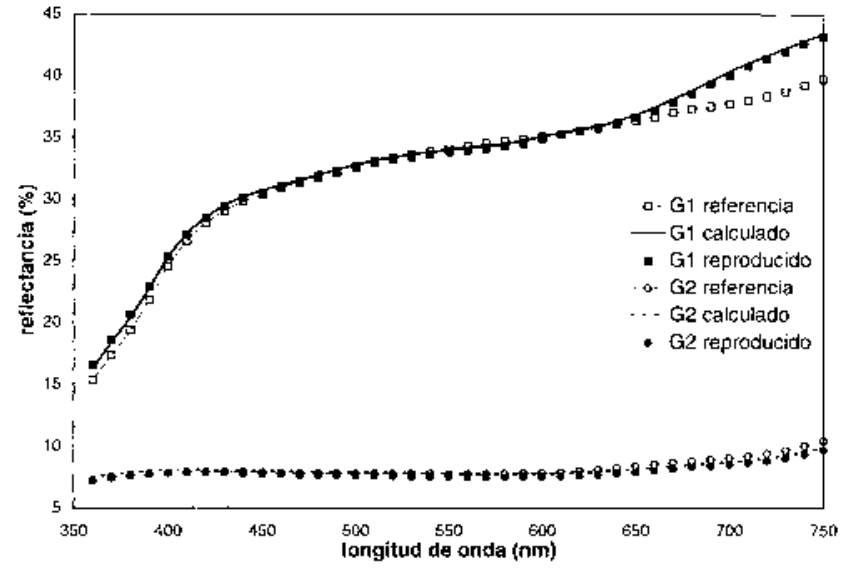

Figura 10. Comparación entre las curvas de reflectancia correspondientes a los productos industriales (G1 y G2 referencia), las calculadas a partir de los valores de $\mathrm{K}$ y S para las composiciones óptimas estimadas para igualarlos a partir del residuo R2 (G1 y G2 calculado), y las correspondientes a la preparación en laboratorio de las composiciones que reproducen los productos industriales (G1 y G2 reproducido).

\section{CONCLUSIONES}

Mediante el procedimiento propuesto, basado en la teoría de Kubelka-Munk, es posible estimar con suficiente precisión las coordenadas cromáticas de una composición de gres porcelánico, obtenida a partir de residuos de producción, y diferentes aditivos. De este modo se puede optimizar el reciclado de los residuos, ya que es posible calcular la composición de la mezcla de residuo y aditivos que posee el color más próximo posible a un modelo de baldosa que se desee producir, $\mathrm{y}$ si dicha diferencia es menor que un umbral previamente establecido, reciclar por completo dicho residuo sin causar distorsiones en los planes de producción. De este modo se evitan los costes de gestión de residuos contaminantes, las pérdidas económicas asociadas al desperdicio de grandes volúmenes de materias primas y se reduce el impacto medioambiental.

\section{BIBLIOGRAFÍA}

1. G. Biffi. Il gres porcellanato: manuale di fabbricazione e tecniche di impiego. Faenza: Faenza Editrice. 1997.

2. R. S. Hunter, R. W. Warold. The measurement of the appearance $2^{\text {nd }}$ edition. John Wiley \& Sons, New York. 1987.

3. Colorimetry $2^{\text {nd }}$ edition. Publication CIE N $N^{\circ} 15.2 .1986$.

4. N. Napier. Colour matching without computers. Ceram. Eng. Sci. Proc. 15[1] 224-232, (1994).

5. P. Kubelka. F. A. Munk. A contribution to the optics of the pigments. Z. Tech. Phys. 12, 593-601, (1931).

6. J. L. Saunderson. Calculation of the color of pigmented plastics. J. Opt. Soc. Am. 32[12] 727-736, (1942).

7. S. H. Murdock, T. D. Wise, R. A. Eppler. Predicting the color of a ceramic glaze. Am. Ceram. Soc. Bull. 69[2], 228-230, (1990).

8. E. D. Campbell, F. W. Billmeyer. Fresnel reflection coefficients for diffuse and collimated light. J. Color. App. 1[2] 39-41, (1971).

9. K. McLaren. The color science of dyes and pigments. Adam Hilger, Bristol. UK. 1986.

10. ASTM.E-308 Standard practice for computing the colors of objects by using the CIE system.

11. H. R. Davidson, H. Hemmendinger. Color prediction using the two-constant turbid-media theory. J. Opt. Soc. Am. 56[8] 1102-1109, (1966). 